\section{Portuguese Journal of Public Health}

\title{
Thanks to Reviewers
}

The Editors of the Portuguese Journal of Public Health would like to extend their gratitude and appreciation to the following reviewers whose comments and criticisms ensured the quality of the papers published.

\author{
Alexandre Vieira Abrantes, Lisbon \\ Maria Joao Aleixo, Almada \\ Ricardo Almendra, Coimbra \\ Cristina Andrade, Tomar \\ Ana Antunes, Lisbon \\ Ricardo Assunção, Lisbon \\ Gonçalo Figueiredo Augusto, Lisbon \\ Catarina Duarte Galhardo Baptista, \\ Lisbon \\ Raquel Camarinha Barbosa, Porto \\ João Brandão, Lisbon \\ Jorge Bravo, Évora \\ Lourdes Bugalho, Lisbon \\ Gisele Câmara, Lisbon \\ Carlos Filipe Afonso de Carvalho, Porto \\ Susana Pinto da Costa, Guimarães \\ Diogo Costa, Bielefeld \\ Teresa Patrone Cotrim, Lisbon \\ Angela Cunha, Aveiro \\ Pedro Delgado, Belfast \\ Carlos Dias, Lisbon \\ Catarina Durao, Lisbon \\ Margarida Eiras, Lisbon \\ Pedro Fidalgo, Lisbon \\ Rita Flores, Lisbon \\ Ana Cristina Fonseca, Faro \\ Inês Fronteira, Lisbon \\ Ana Gama, Lisbon \\ Antonio Gerbase, Geneva \\ Ana Rita Goes, Lisbon \\ Bernardo Gomes, Santa Maria da Feira \\ Judite Gonçalves, Lisbon \\ Manuel Gonçalves-Pereira, Lisbon \\ Miguel Gouveia, Lisbon \\ Luís Graça, Lisbon \\ Bruno Heleno, Lisbon \\ Musa Abubakar Kana, Kaduna \\ Pedro A. Laires, Lisbon \\ Ines Leal, Lisbon \\ Teresa Leão, Lisbon
}

Liz Leese, Buxton

Andreia Leite, Lisbon

Ana Rita Londral, Lisbon

Silvia Lopes, Lisbon

Isabel Loureiro, Lisbon

Carlota Louro, Lisbon

Raquel Lucas, Porto

Rita Macedo, Lisbon

Ana Paula Almeida Martins, Lisbon

Marta Mello e Sampayo, Lisbon

Lucio Meneses de Almeida, Coimbra

Helder Mota-Filipe, Lisbon

Helena Nogueira, Coimbra

Olena Radomska Oliveira, Braga

Carlos A.O. Pavao, Atlanta, GA

Frederico Peres, Rio de Janeiro

Diogo Pires, Alcabideche

Sónia Pós de Mina, Leiria

João Filipe Raposo, Lisbon

Sofia Ravara, Covilhã

Manuel Castro Ribeiro, Lisbon

Raquel Esaguy Rodrigues, Lisbon

Rafaela Rosário, Minho

Ana Russo, Lisbon

Teresa Sancho, Faro

Rui Santana, Lisbon

Ana João Santos, Lisbon

Alexandre Sepriano, Leiden

Florentino Serranheira, Lisbon

Maria Vania Rocha Silva Nunes, Lisbon

José Augusto Simões, Covilhã

Patrícia Soares, Lisbon

Jose Mira Solves, Alicante

Joana Sousa, Lisbon

Paulo Sousa, Lisbon

Mafalda Sousa Uva, Lisbon

Dora Vaz, Amadora

Carla Viegas, Lisbon

Susana Viegas, Lisbon

Daniel Virella, Lisbon (c) 2021 The Author(s). Published by S. Karger AG, Basel on behalf of Escola Nacional de Saúde Pública

This is an Open Access article licensed under the Creative Commons Attribution-NonCommercial-4.0 International License (CC BY-NC) (http://www.karger.com/Services/OpenAccessLicense), applicable to the online version of the article only. Usage and distribution for commercial purposes requires written permission. 